\title{
A Dual-Mode Actinic EUV Mask Inspection Tool
}

Y. Liu, A. Barty, E. Gullikson, J. S.Taylor, J. A. Liddle, O. Wood

March 23, 2005

SPIE Microlithography

San Jose, CA, United States

February 2, 2005 through April 3, 2005 
This document was prepared as an account of work sponsored by an agency of the United States Government. Neither the United States Government nor the University of California nor any of their employees, makes any warranty, express or implied, or assumes any legal liability or responsibility for the accuracy, completeness, or usefulness of any information, apparatus, product, or process disclosed, or represents that its use would not infringe privately owned rights. Reference herein to any specific commercial product, process, or service by trade name, trademark, manufacturer, or otherwise, does not necessarily constitute or imply its endorsement, recommendation, or favoring by the United States Government or the University of California. The views and opinions of authors expressed herein do not necessarily state or reflect those of the United States Government or the University of California, and shall not be used for advertising or product endorsement purposes. 


\title{
A Dual-Mode Actinic EUV Mask Inspection Tool
}

\author{
Yanwei Liu ${ }^{1}$, Anton Barty ${ }^{2}$, Eric Gullikson ${ }^{1}$, John S.Taylor ${ }^{2}$, J. Alexander Liddle ${ }^{1}$ and Obert Wood $^{3}$ \\ ${ }^{1}$ Center for X-ray Optics, Lawrence Berkeley National Laboratory, Berkeley, CA 94720 \\ ${ }^{2}$ Lawrence Livermore National Laboratory, P.O. Box 808, Livermore, CA 94550 \\ ${ }^{3}$ SEMATECH, 255 Fuller Road, Suite 309, Albany, NY 12203
}

\begin{abstract}
To qualify the performance of non-actinic inspection tools, a novel EUV mask inspection system has been installed at the Advanced Light Source (ALS) synchrotron facility at Lawrence Berkeley National Laboratory. Similar to the older generation actinic mask inspection tool ${ }^{1}$, the new system can operate in scanning mode, when mask blanks are scanned for defects using 13.5-nm in-band radiation to identify and map all locations on the mask that scatter a significant amount of EUV light. By modifying and optimizing beamline optics (11.3.2 at ALS) and replacing K-B focusing mirrors with a high quality Schwarzschild illuminator, the new system achieves an order of magnitude improvement on in-band EUV flux density at the mask, enabling faster scanning speed and higher sensitivity to smaller defects. Moreover, the system can also operate in imaging mode, when it becomes a zone-plate-based full-field EUV microscope with spatial resolution better than $100 \mathrm{~nm}$. The microscope utilizes an off-axis setup, making it possible to obtain bright field images over a field-of-view of $5 \times 5 \mu \mathrm{m}^{2}$.
\end{abstract}

Keywords: extreme ultraviolet lithography; mask; reticle; defect; inspection; microscopy.

\section{MULTILAYER DEFECT INSPECTION REQUIREMENTS}

A defect is any area on the mask that causes unwanted variations in EUV dose in the aerial image at the wafer plane of a lithographic exposure tool. For reflective EUV masks, defects can occur in both the reflective multilayer coating and the patterned absorber layer. Defects in the patterned absorber layer consist of regions absorber material is unintentionally added or removed from the pattern leading to errors in the reflected field. There currently exists a mature technology for inspecting and repairing defects in the mask pattern. However, a problem unique to EUVL is the existence of defects in the reflective multilayer coating applied to mask blanks prior to patterning. These defects can perturb the reflected field, causing unwanted variations in aerial image contrast, and manifest themselves as defects in the printed image.

A typical EUV multilayer coating consists of 40-80 bilayers of molybdenum and amorphous silicon with each bilayer consisting of approximately $3 \mathrm{~nm}$ Mo and $4 \mathrm{~nm} \mathrm{Si}$. The reflectivity of the multilayer is a resonant property of the alternating layer structure and is at an optimum when all the layers interfere constructively satisfying the Bragg condition; because reflection takes place throughout the bulk of the multilayer, any deformation or disruption of the layer structure can manifest itself as a defect. Ideally, the goal is to produce defect-free mask blanks; however this may be difficult to achieve in practice. It is therefore essential to have strategies for inspecting mask blanks prior to patterning in order to determine whether there are any defects present on the mask blank. It is anticipated that high-throughput visible-light inspection tools will be used for this purpose in a production environment; however the defects are only critical if they manifest themselves in the reflected EUV light. Therefore, it is necessary to determine whether there are any defects observed during inspection with EUV light that are not being detected by the visible light inspection tools in order to have confidence in the visible light inspection strategy. The purpose of the actinic inspection tool is to help answer this question. 
The goal of this project is to design an actinic mask inspection tool capable of operating in two modes: high-speed scanning for the detection of multilayer defects (inspection mode), and a high-resolution aerial image mode in which the image emulates the imaging and illumination conditions of a stepper system (aerial image or AIM mode). The purpose and objective of these two modes is as follows:

\section{Defect inspection mode}

Defect inspection mode is designed for scanning large areas of the mask for defects on or in EUV multilayer coatings, regardless of whether they are particles on the surface, were caused during coating or were present on the substrate prior to coating. The goal is to detect the presence of anomalies on a mask blank and to store the co-ordinates for subsequent review in AIM mode, thus it is not essential that the illumination and imaging conditions match that of a production stepper. Determining whether or not the anomalies are "printable defects" will be determined during a subsequent defect review step. Potential uses for this imaging mode include:

- Correlating the results obtained using actinic inspection with results obtained using other non-EUV defect inspection systems to verify that the non-EUV scanning systems are detecting all critical defects;

- Gaining sufficient information to associate defects with particular processes, such as various stages of multilayer deposition, shipping, handling, etc; and

- $\quad$ Assessing the density and EUV impact of surface and multilayer anomalies

Because of the low defect density achieved using current multilayer coating technology, it is necessary to be able to efficiently scan large areas of the mask in order to obtain sufficient statistics for use in cross-correlation experiments. Speed of operation as well as sensitivity is therefore key to operation in defect inspection mode.

\section{Aerial Image Microscope (AIM) mode}

In AIM mode, the tool is configured so that the collected data emulates the aerial image of a stepper system, thereby enabling rapid evaluation of whether or not anomalies identified during inspection are printable defects and without the need for a resist exposure step. The main uses of the microscope in this mode would be:

- Review of multilayer and pattern defects to determine their printability

- Defect review following a repair process to assess the success of the operation

- Investigation of the effects of illumination and NA on the printed image

- $\quad$ Process window analysis of defects and other mask features

- Characterising defects on both patterned and unpatterned masks (i.e. blanks)

An essential characteristic of operation in this mode is that the illumination and imaging conditions, including scanning through focus, should emulate as accurately as possible that of a production stepper system. This mode is designed for local review of a relatively small number of defects; therefore it is not necessary to have the same high-speed throughput required for defect inspection mode.

\section{DUAL-MODE INSPECTION SYSTEM DESIGN}

The inspection system must be able to inspect and image defects on both blank and patterned masks produced using the same industrial processes as intended for volume manufacture. It is therefore essential that the inspection system be compatible with masks used in both the first generation production tools and pre-production tools such as the EUV Micro-Exposure Tool (MET). The inspection tool specifications are therefore driven by a combination of anticipated production stepper specifications, and the practical requirements of both high-speed and AIM mode operation.

\subsection{High-speed scanning mode}

For high-speed scanning mode, the goal is to scan a large area of unpatterned mask blank looking for defects in the multilayer coating. Speed is essential, as the defect density is anticipated to be low - of the order of 0.05 defects $/ \mathrm{cm}^{2}$ or one defect in every $20 \mathrm{~cm}^{2}$ (on average). In order to obtain sufficient statistics to determine whether visible light scattering tools are seeing all relevant multilayer defects, it is necessary to scan several hundred square centimeters of mask blank and to be able to cross-register the locations of observed potential defects between different tools. The capacity for high-speed, continuous operation of the tool over large areas of mask surface is therefore critical. 
To date the highest sensitivity for EUV defect detection has been obtained in dark-field mode where only the light scattered out of the entrance pupil is detected. There is background scatter from the multilayer of the order of $0.5 \%$ to $1 \%$ above which the dark-field signal from a defect must rise in order to be registered as a defect - and this sets a theoretical bound on the signal-to-noise ratio obtainable in dark-field imaging mode. Signal-to-noise improves as the scanning spot size is decreased, at the expense of reduced flux and more pixels required to span a complete mask. Our analyses of the trade-offs between scan speed, beamline flux and signal-to-noise levels for this system indicates an optimum point with a $1 \mu \mathrm{m}$ spot size on the mask. Dark-field imaging is relatively insensitive to absorption-only defects, therefore we include the capacity for simultaneous bright-field and dark-field detection in scanning mode. All data is collected in real time and saved for later analysis.

The conceptual layout of the system is illustrated below. A $1 \mu \mathrm{m}$ spot is produced by re-imaging a $20 \mu \mathrm{m}$ pinhole onto the mask using Schwarzschild optics with a demagnification factor of 20x. Because of space constraints between the Schwarzschild optic and the mask it difficult to put detectors in this location; instead, we use a scraper mirror to reflect the scattered light to the side where the brightfield signal is captured on a photodiode and the darkfield signal is registered on a channelplate.

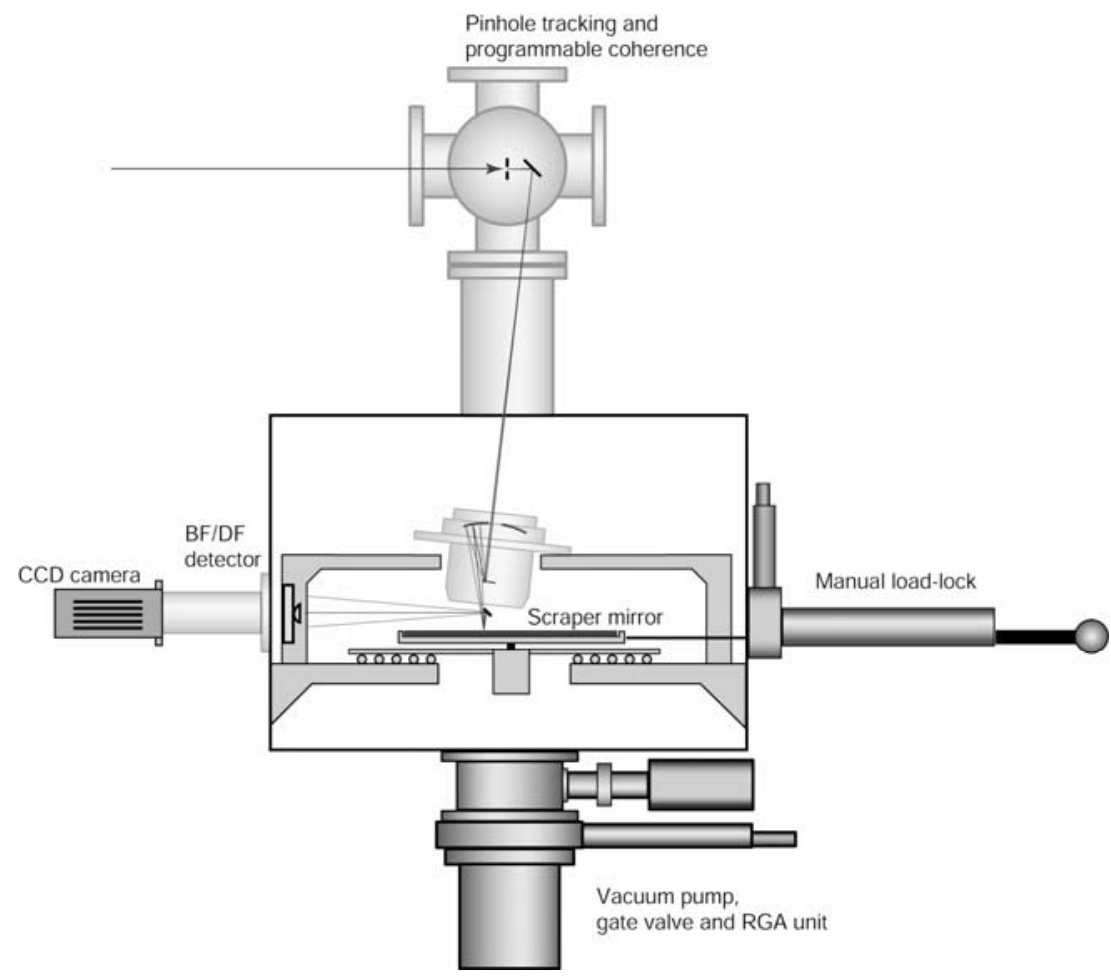

Figure 1

Conceptual layout of the scanning system design. A $1 \mu \mathrm{m}$ spot is produced by re-imaging a $20 \mu \mathrm{m}$ pinhole onto the mask using Schwarzschild optics with a demagnification factor of 20x. Because of space constraints between the Schwarzschild optic and the mask, it is difficult to put detectors in this location; instead, we use a scraper mirror to reflect the scattered light to the side where the brightfield signal is captured on a photodiode and the darkfield signal is registered on a channelplate.

The EUV light incident into the $1 \mu \mathrm{m}$ spot on the mask has been measured and characterized. Just prior to the mask, the photodiode current was measured to be $1.6 \mathrm{e}-7 \mathrm{Amp}$ at 230mA ALS current. The diode response is known to be $0.12 \mathrm{~A} / \mathrm{W}$ at EUV wavelengths, giving an incident flux on the mask of $1.3 \mu \mathrm{W}$ of in-band EUV light centered on $13.4 \mathrm{~nm}$ within the bandpass. This is well within specifications for achieving the target $2 \mathrm{~cm}^{2} / \mathrm{hr}$ scan speed of the mask scanner in high speed scanning mode. 


\subsection{Imaging mode}

In imaging mode the tool is used to capture images of defects and to provide data on defect printability. The results obtained by measuring the aerial image should, as far as practically possible, provide the same aerial image information that would be obtained in a stepper system. Mimicking the performance of a stepper, albeit over a smaller field, is therefore essential if the results obtained using an aerial image microscope are to provide a reliable assessment of defect printability.

Equivalence of the through-focus aerial image performance with that of a production system is essential, as many types of defects manifest themselves as changes in the aerial image as a function of focal position. Manufacturing aberrations affect both in-focus and through-focus behavior, and therefore impact the aerial image. A previous design study found that the best way to achieve this equivalence is through the fabrication of a custom EUV optic specifically designed for aerial image measurement ${ }^{2}$, and commercial AIM systems with these requirements are in production for use in industry ${ }^{3}$. For the purposes of this project it not justified to construct a custom optic capable of meeting full equivalence with the imaging quality of a stepper optic; however, it is possible to emulate the essential properties of numerical aperture and aberration control at lower cost using a zone plate optic.

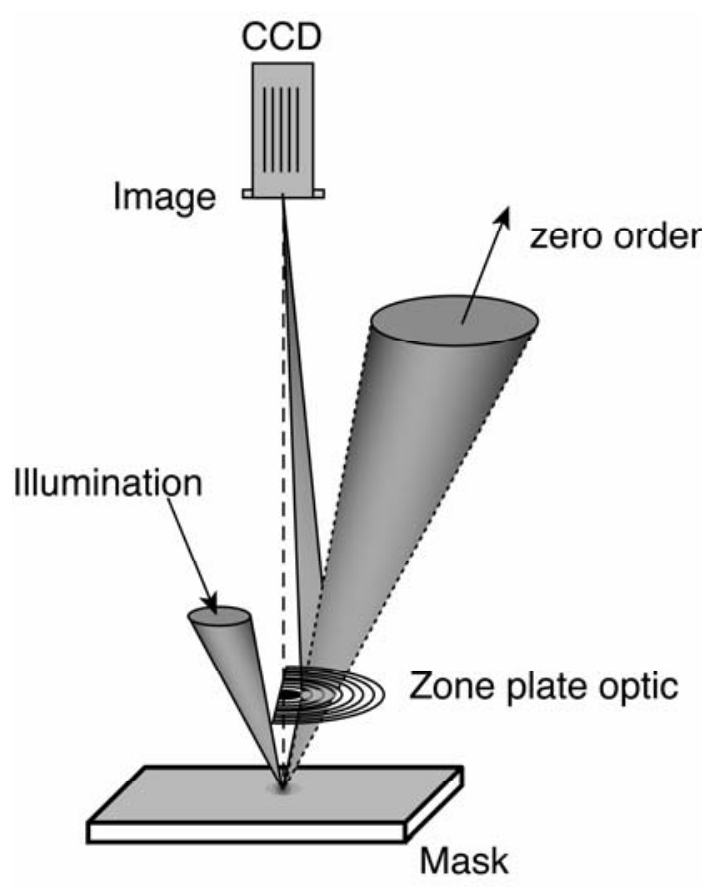

Figure 2

Schematic layout of the zone plate imaging system. An off-axis potion of a $0.166 \mathrm{NA}$ parent zone plate is used to image the mask onto the CCD camera, keeping the whole field-of-view in focus and eliminating keystone distortion. To illuminate the mask, only that portion of the zone plate involved in imaging the mask is fabricated - the remainder is left unpatterned and may be used to introduce illumination into the system. Using the off-axis design has the added benefit of physically separating unwanted diffraction orders from the zone plate optic. Due to space constraints within the system, a fold mirror (not shown) is included in our imaging system to project the image onto a CCD to the side of the mask.

The imaging mode subsystem of the EUV mask inspection tool uses a zone plate optic to produce a real, magnified image of the mask on a CCD camera. In order to properly emulate the imaging characteristics of a $0.25 \mathrm{NA} 4 \mathrm{x}$ reduction lithography stepper it is necessary to have a pupil with a clear aperture of $0.0625 \mathrm{NA}$ observing the mask at an angle of incidence of $6^{\circ}$. In this way the zone plate optic will see the mask with the same conditions (angle of incidence, NA, wavelength and illumination) as the projection optics in the lithographic system, and should therefore provide an image that is essentially similar to that anticipated when the mask is printed in a lithographic system. 
Zone plate microscopes typically operate in transmission mode with the condenser and imaging optics on opposite sides of the object. This relies on the object being transmissive, whilst EUV masks are reflective. In order to image a reflective EUV mask, it is necessary to fold the system back on itself so that both the illumination and imaging optics are on the same side of the object. The desired mask-side aperture of $0.0625 \mathrm{NA}$ at $6^{\circ}$ allows adequate clearance for both the optics and illumination on the same side of the mask, although space is constrained. These issues are similar to accommodating illuminator clearance when designing the mask-side of lithographic optics.

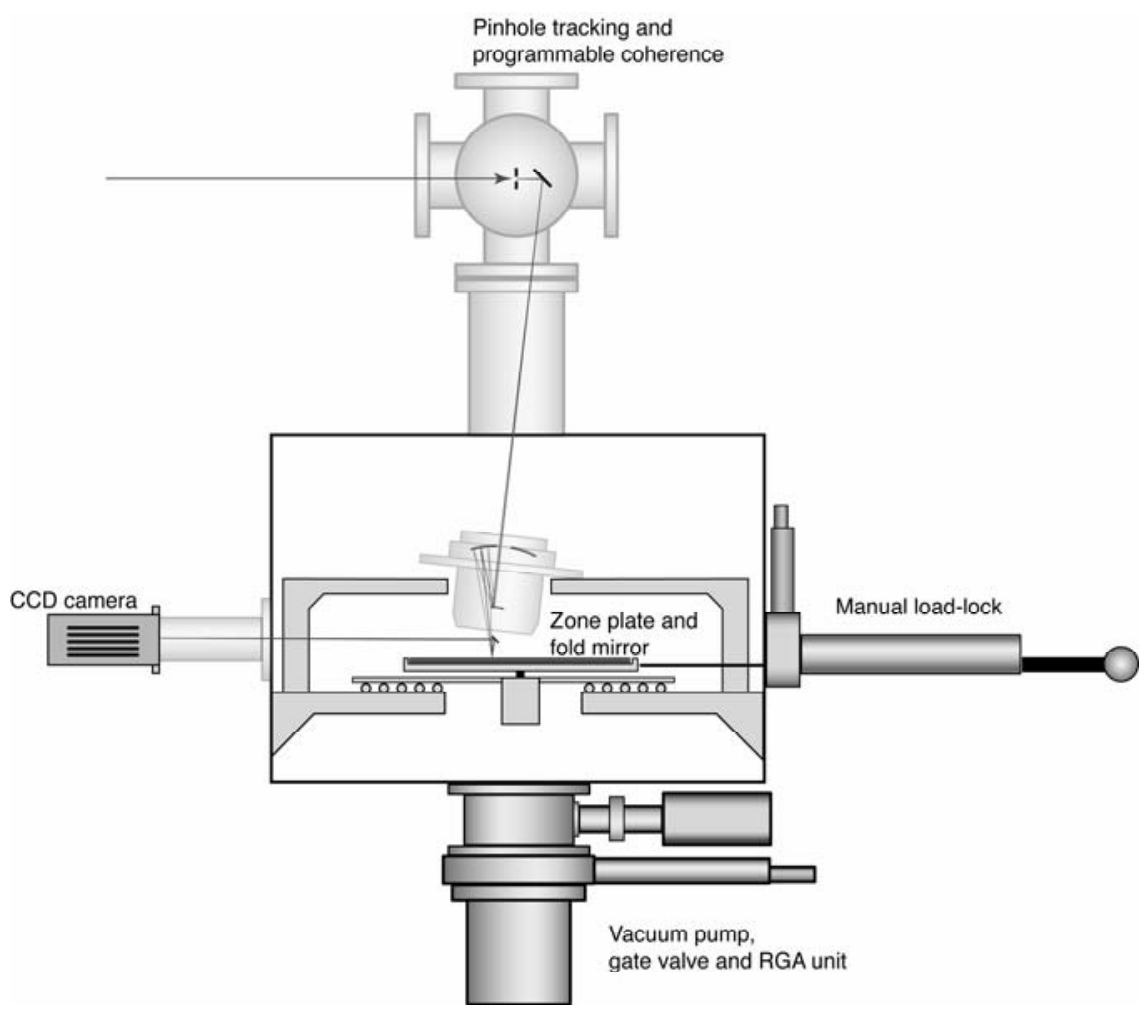

Figure 3

Defect inspection system modified for operation in high-resolution imaging mode. The scraper mirror is replaced with a reflective zone plate lens, with an image of the mask being formed on an EUV CCD camera located to the side of the chamber. Note the inclusion of a fold mirror to redirect the image to the side of the mask. This is necessary due to space constraints with locating the imaging system beneath the Schwarzschild optics.

The simplest zone plate design contemplates a single imaging element (zone plate) imaging the mask with a $0.0625 \mathrm{NA}$ zone plate tilted with respect to the mask so that the optical axis of the imaging system is at $6^{\circ}$ to the mask. The imaging system is therefore a tilted optical system and as such will suffer from keystone distortion and require a tilted image plane. For a single zone-plate lens the resultant image will suffer from distortion, and it will be difficult to maintain the whole field of view in focus at the same time.

An alternative design that can correct for the effects of imaging the mask at an angle is shown in Figure 2. By using an off-axis portion of an on-axis zone plate, the image is formed on an axis perpendicular to the mask. In order to capture the marginal ray of an off-centre $0.0625 \mathrm{NA}$ AIM pupil centered on a chief ray angled at $6^{\circ}$ to the mask it is necessary to have a parent zone plate with a numerical aperture of $0.166 \mathrm{NA}$.

This design has the benefit of eliminating keystone distortion suffered by the tilted optic design, and results in object and image planes parallel to one another and the zone plate. A second advantage of adopting this design is that the zero and 
first order diffraction orders are physically separated, reducing flare in the system and allowing for more relaxed placement of the order sorting aperture (if required). A schematic of the layout of the patterned zone plate is shown in Figure 4.

The design of a zone plate is completely specified by the wavelength, bandwidth and resolution (NA) of the optic. For AIM mode imaging the NA of the optic is fixed and the wavelength is set at $13.4 \mathrm{~nm}$ by the illumination optics in the scanning system, leaving bandwidth as the only undetermined parameter. The allowable bandwidth for a zone plate is set by restrictions on the allowable chromatic aberration in the imaging system, and results from the fact that zone plates are a highly dispersive optic ${ }^{4}$.

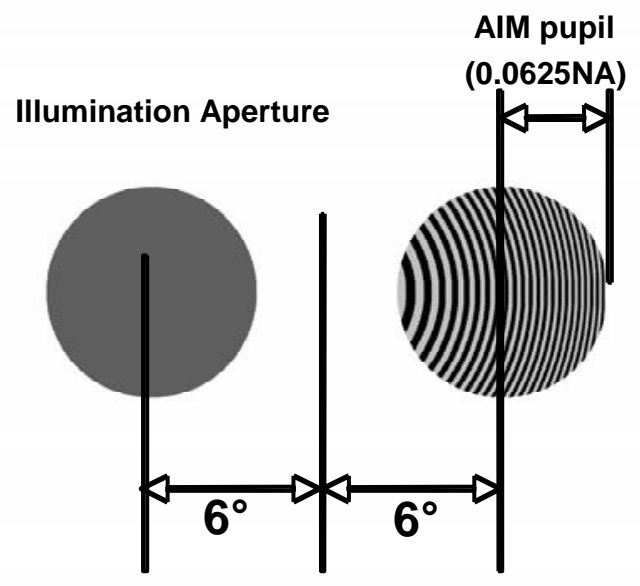

Figure 4

Schematic of the off-axis zone plate optic design. The clear aperture on the left is used to introduce illumination to the mask, whilst the zone plate structure on the right is used to forms the image of light reflected from the mask.

In order to have a workable zone plate design we chose to operate in the AIM mode with the beamline monochromator in place, providing between $0.05 \%$ and $0.1 \%$ illumination bandwidth, depending on exit slit settings. This enables the use of up to 2000 zones in the zone plate producing a focal length close to $1 \mathrm{~mm}$. This enables the zone plate to be held $1 \mathrm{~mm}$ from the mask, reducing the risk of accidental contact with the mask surface. Based on the desired wavelength, and the design constraints of an AIM mode imaging objective, key zone plate design parameters are:

$\begin{array}{ll}\text { Parent NA: } & 0.166 \mathrm{NA} \\ \text { Outer zone width: } & 40 \mathrm{~nm} \\ \text { Number of zones: } & 2000 \\ \text { Focal length } & 0.977 \mathrm{~mm} \\ \text { Parent diameter: } & 325 \mu \mathrm{m} \\ \text { Useable field: } & 108 \mu \mathrm{m} \\ \text { Working distance: } & 0.97 \mathrm{~mm} \\ \text { Track length: } & 0.5 \mathrm{~m} \text { for } 500 \mathrm{x} \text { magnification }\end{array}$

Zone plates corresponding to the above design were fabricated in the CXRO nanofabrication laboratory at Lawrence Berkeley National Laboratory. Two sets of zone plates were fabricated, one set on a transmissive silicon nitride membrane comprising portions of circular arcs as illustrated above, whilst a second set of free-standing zone plates also contains supportive spokes. The silicon nitride membrane absorbs EUV light, so there is loss in throughput associated 
with patterning on a transmissive membrane. The free standing zone plates are more fragile and more susceptible to deformation due to stress in the nickel plating, but offer a significantly higher throughput.

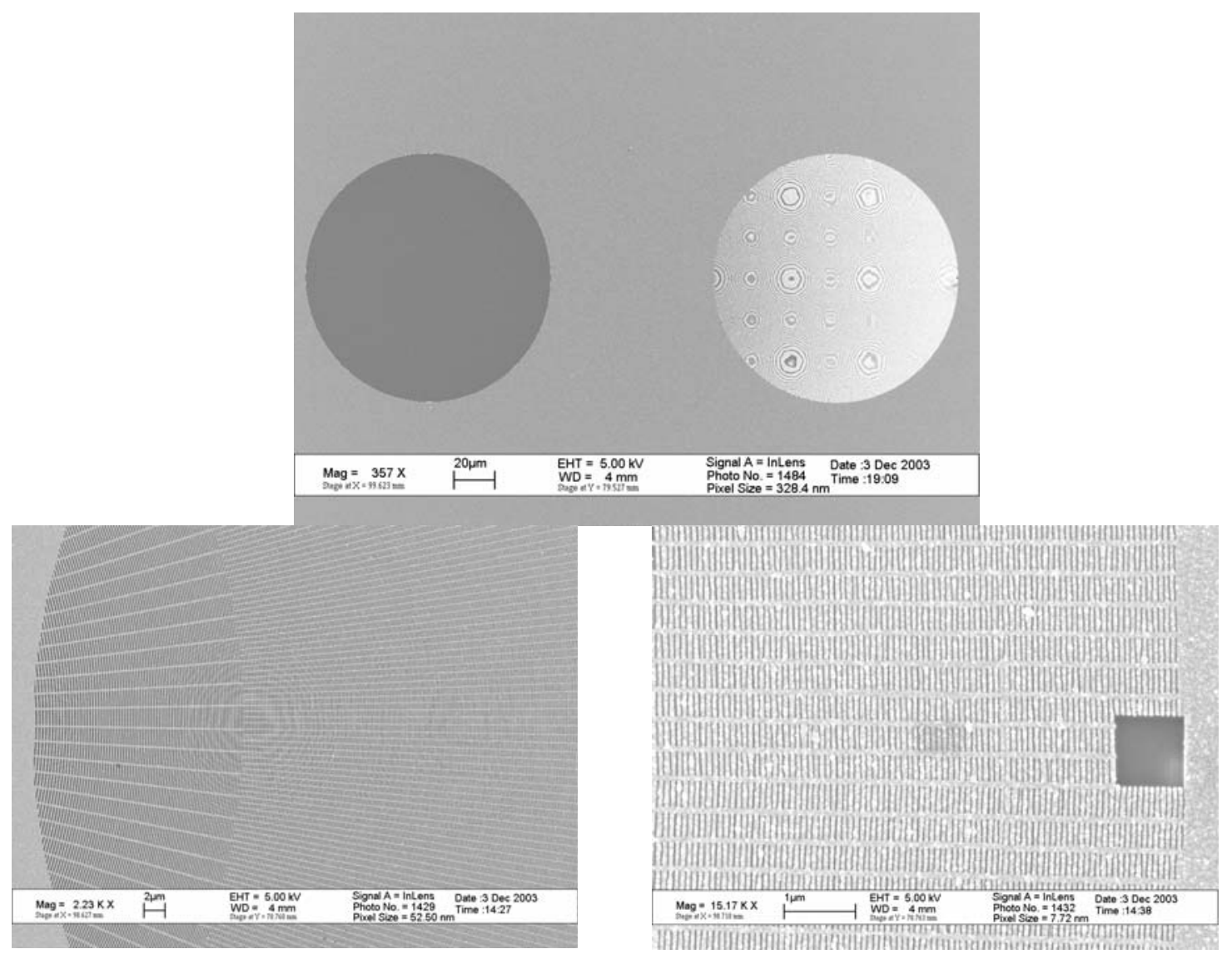

Figure 5

SEM image of free-standing zone plate at low magnification (top) showing both the illumination and imaging apertures, and at higher resolution (below). Note the web of support structures required in order to maintain the free-standing pattern. Inner and outer edges of the zone plate pattern seen at higher magnification. Width of the outermost zones is $40 \mathrm{~nm}$.

\section{DEMONSTRATION OF IMAGING PERFORMANCE}

In this section we demonstrate imaging performance and resolution obtained with the free standing zone plate by imaging a test pattern of dense line-space features near the resolution cutoff of this optic.

In this configuration the distance from the zone plate to the CCD is on the order of $650 \mathrm{~mm}$. The total magnification is approximately 650x, given the zone plate focal length of approximately $1 \mathrm{~mm}$. The CCD was a scientific-grade Princeton Instruments CCD with $1024 \times 1024$ array of $24 \mu \mathrm{m}$ pixels and a digitisation depth of 12 bits, giving an effective pixel size on the object of $40 \mathrm{~nm}$ and a data range from 0 to 4096 counts per pixel. Data was collected from a test pattern consisting of several features, including dense line-space patterns near the resolution cutoff of this optic. 


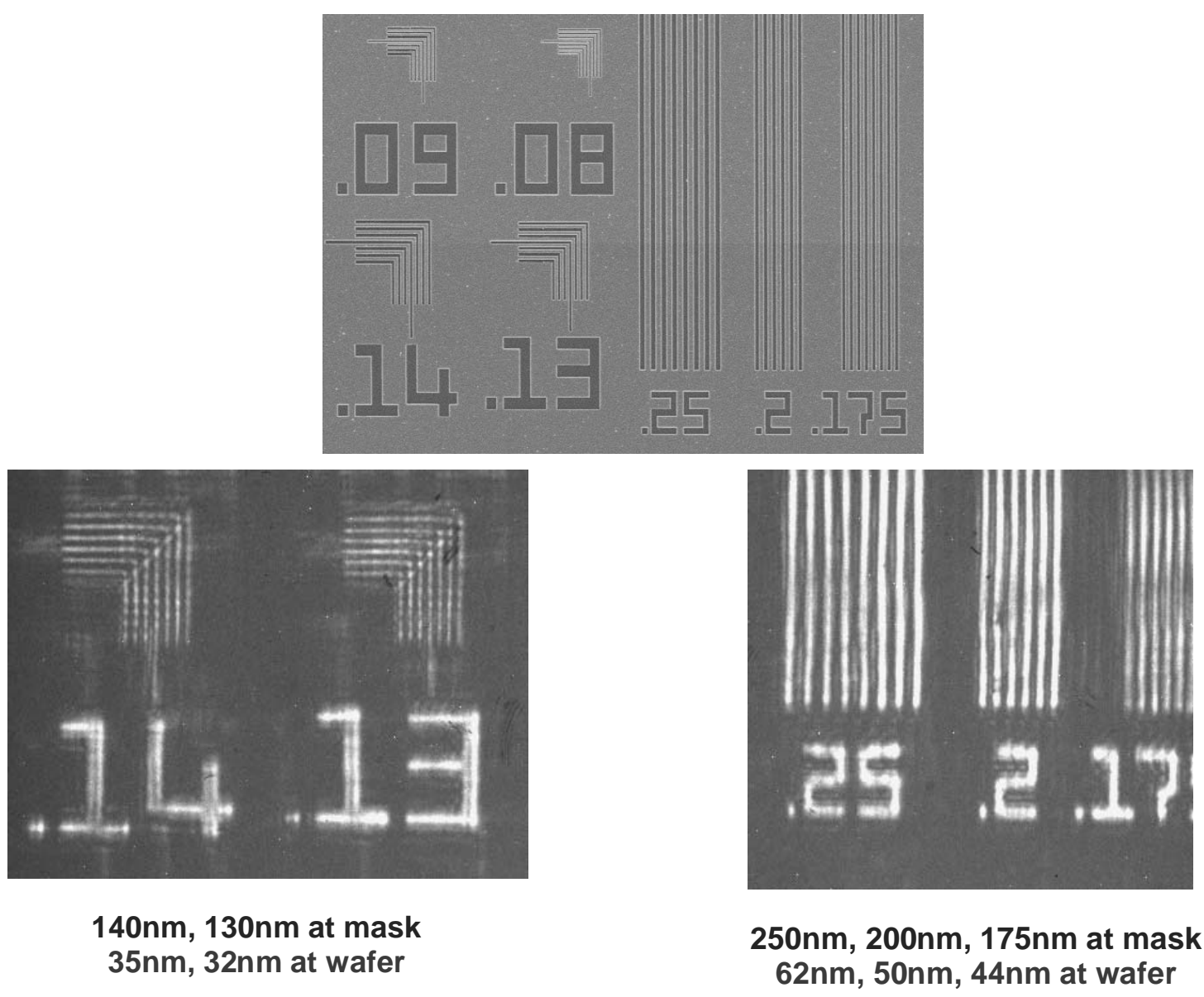

Figure 6

SEM image of a portion of the test pattern used in the imaging tests (top) and sample EUV images collected on the CCD using the zone plate microscope (below)

As previously described, the clear numerical aperture facing the mask is 0.0625NA to emulate the mask-side resolution of a $0.25 \mathrm{NA}, 4 \mathrm{x}$ reduction stepper. This gives a diffraction-limited Rayleigh resolution of $107 \mathrm{~nm}$ for the optic assuming coherent illumination and a perfect sinusoidal grating object. Figure 7 shows the EUV image formed on the CCD camera by the zone plate of a portion of the test pattern consisting of features near the resolution cutoff of the zone plate optic. Note that $110 \mathrm{~nm}$ lines are clearly resolved and that features down to $80 \mathrm{~nm}$ on the mask (20nm printed at the wafer) are clearly visible.

A line-out section through this data is shown in Figure 8 clearly demonstrates a resolution of $110 \mathrm{~nm}$ features on the mask, with features below $80 \mathrm{~nm}$ on the mask also clearly resolved with somewhat lower contrast. The ability to resolve features smaller than the Rayleigh limit, and the observed decrease in contrast, is qualitatively consistent with the contrast roll-off expected from partially-coherent illumination. 


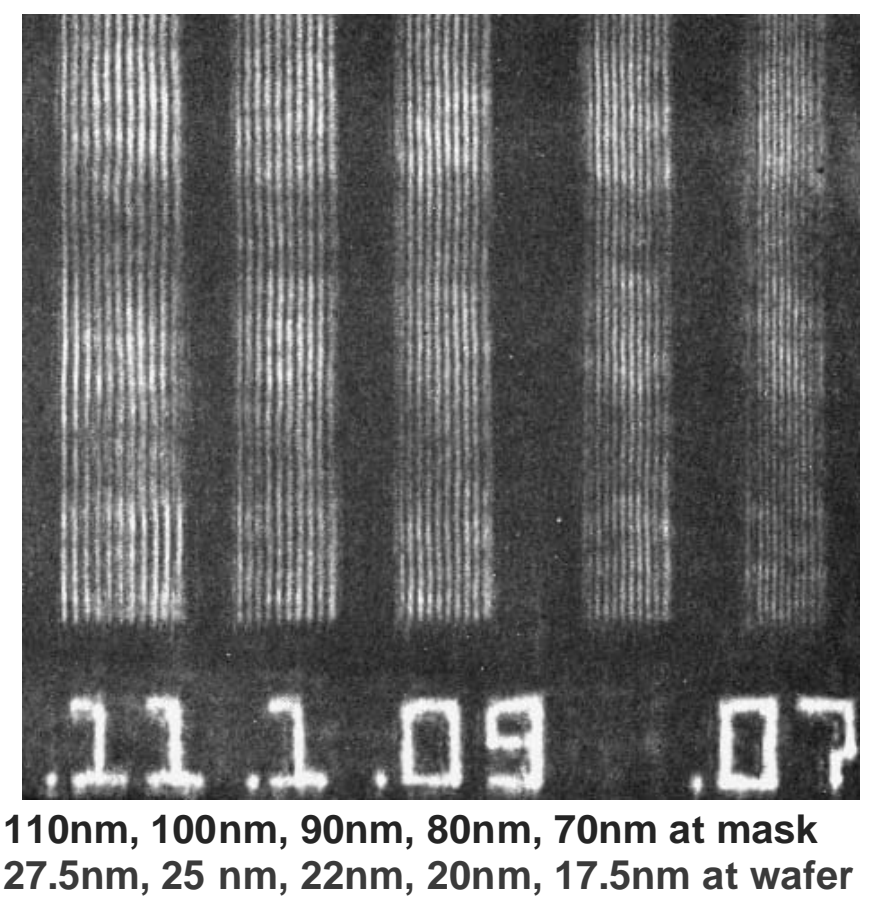

Figure 7

Digital EUV image collected using the zone plate optic of 1:1 dense line space patterns ranging in size from 110nm to 70nm.

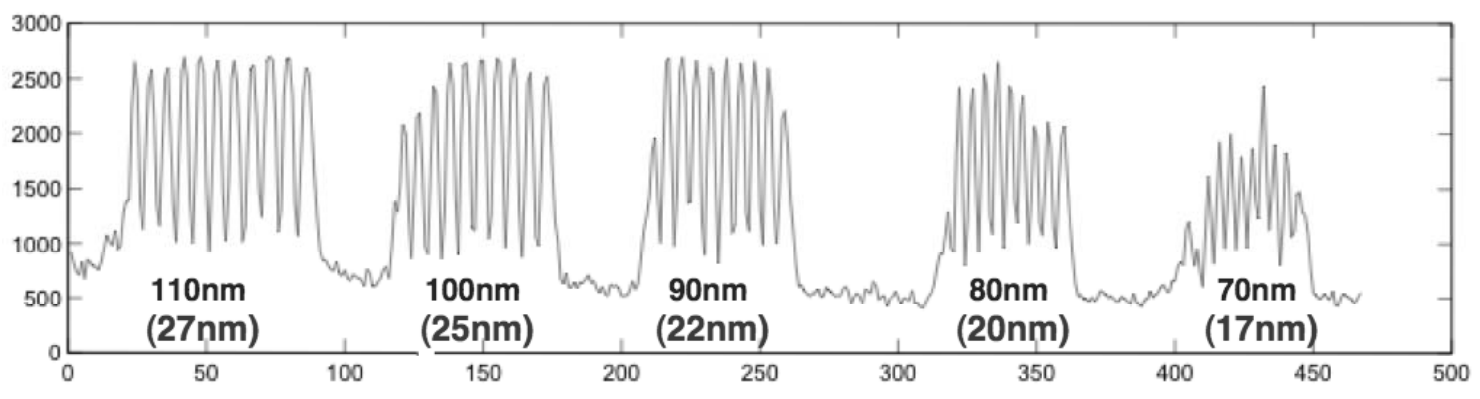

Figure 8

Line-out across the data shown in Figure 7 demonstrating the contrast achieved at 110nm and smaller features on the mask. Labels correspond to feature size on the mask, whilst the numbers in parentheses are the corresponding printed linewidth at the wafer with $4 \mathrm{x}$ reduction. Inspection of this data shows that $110 \mathrm{~nm}$ features are well resolved, with resolution still acceptable at $80 \mathrm{~nm}$ features. If printed in a $4 \mathrm{x}$ reduction stepper, this corresponds to clearly resolved $27 \mathrm{~nm}$ printed lines at the wafer, with the optic resolution extending to below $20 \mathrm{~nm}$ printed lines at the wafer.

SUMMARY

We have performed resolution tests of the zone plate optic used in the EUV mask inspection system installed at beamline 11.3.2 at the ALS and have shown that both the irradiance of EUV light in the final system is at or above that predicted in the initial throughput budget, and that initial optic performance is capable of clearly resolving $80 \mathrm{~nm}$ features on the mask (20nm on the wafer in a $4 \mathrm{x}$ stepper system) using a zone plate optic. 


\section{REFERENCES}

1. S. Jeong et al, “Actinic EUVL mask blank defect inspection system”, Proc. SPIE Vol. 3676, p. 298-308, 1999.

2. A. Barty, J.S.Taylor, R.Hudyma, E.Spiller, D.W.Sweeney, G.Shelden and J-P Urbach, "Aerial image microscopes for the inspection of defects in EUV masks" (2002) 1889 Proc. SPIE 1073.

3. A. Brunton, et. al., "High resolution EUV imaging tools for resist exposure and aerial imaging monitoring", Paper 5751-06 presented at Emerging Lithographic Technologies IX, SPIE, San Jose, CA March 1-3, 2005.

4. A. G. Michette, Optical Systems for Soft X-Rays, Plenum, NY 1986.

This work was performed under the auspices of the U. S. Department of Energy by University of California, Lawrence Livermore National Laboratory under contract No. W-7405-Eng-48. 